\section{PS2:37 ANTI-SMITH ANTIBODIES INFLUENCE ON CLINICAL, BIOLOGICAL AND IMMUNOLOGICAL FEATURES OF SYSTEMIC LUPUS ERYTHEMATOSUS IN TUNISIAN PATIENTS}

T Ben Salem, M Tougorti, I Naceur, I Ben Ghorbel, M Lamloum, MH Houman. Department of Internal Medicine, La Rabta University Hospital, Tunis, Tunisia

\subsection{6/lupus-2018-abstract.85}

Backdround Anti-Smith $(\mathrm{Sm})$ antibodies are highly specific for systemic lupus erythematosus (SLE), their associations with some organ involvements were described.

Objectives The aim of our study was to determine clinical, biological and immunological characteristics in SLE patients according to the presence of anti-Sm antibodies.

Methods A retrospective study of a group of Tunisian patients with SLE (fulfilling the ACR revised criteria) was conducted in an internal medicine department. Patients were divided in two groups according to the presence (groupe1) or not (group 2) of anti-Sm antibodies. Variables with a p inferior to 0.05 were considered to be statistically significant.

Results Anti-Sm antibodies were screened in 153 among 246 SLE patients and were positive in $64 \%$ of patients. Mean age at SLE diagnosis was significantly lower in the group 1 (31.82 years \pm 12.70 vs 36.16 years $\pm 12.34 ; p=0.043$ ). Poor general state was the only clinical feature significantly more frequent in group 1 (54.2\% vs $36.5 \%$; $=0.04)$. Lupus nephritis was more frequent in patients with anti-Sm antibodies without significant difference $(47.9 \%$ vs $38.2 \% ; \mathrm{p}=0.24)$. Arthritis were more frequent in group $2(42.6 \%$ vs $50 \% ; p=0.38)$. Central nervous system manifestations $(16.7 \%$ vs $16.4 \%)$, pericarditis $(36.1 \%$ vs $31.4 \%)$ and pleural effusion $(23.4 \%$ vs $27.8 \%)$ were similar in the two groups. Anaemia $(77.1 \%$ vs $74.5 ; p=0.7)$, leucopenia ( $49 \%$ vs $47.2 \%$; $=0.8$ ) and thrombocytopenia $(26 \%$ vs $15 \%$; $=0.12)$ were more frequent in patients with anti-Sm antidodies without significant differences. Anti-Sm antibodies were significantly associated with anti-RNP antibodies (78.6\% vs $17.6 \%$; $<<0.0001)$. Remission was less frequent is group $1(70.4 \%$ vs $82.9 \% ; \mathrm{p}=0.14)$ and death was more frequent in this group $(16 \%$ vs $10.3 \%$; $=0.4)$ without significant differences.

Conclusions In our patients, anti-Sm antibodies prevalence was higher than those reported in other cohorts (5\% to 49\%). Patients with anti- $\mathrm{Sm}$ antibodies seem to develop clinical SLE manifestations earlier than other patients. In our study, antiSm antibodies doesn't influence SLE clinical course and weren't associated with hematologic disorders, whereas in another series, anti-Sm antibodies were associated with lupus nephritis, serositis, anaemia and leucopenia.

\section{PS2:38 COMPARATIVE TISSUE TRANSCRIPTOME ANALYSIS BY NEXT-GENERATION SEQUENCING REVEALS NOVEL PATHWAYS THAT CHARACTERISE GENETIC SUSCEPTIBILITY AND DEVELOPMENTAL BIOLOGY IN SYSTEMIC LUPUS ERYTHEMATOSUS (SLE)}

\footnotetext{
${ }^{1,2}$ E Frangou, ${ }^{1} \mathrm{M}$ Grigorioy, ${ }^{1} \mathrm{~A}$ Banos, ${ }^{3} \mathrm{G}$ Bertsias, ${ }^{1,4} \mathrm{E}$ Dermitzakis, ${ }^{1,2,5} \mathrm{DT}$ Boumpas. ${ }^{1}$ Biomedical Research Foundation of the Academy of Athens, Greece; ${ }^{2}$ Medical School, University of Cyprus, Nicosia, Cyprus; ${ }^{3}$ Medical School, University of Crete, Heraklion, Greece; ${ }^{4}$ Medical School, University of Geneva, Switzerland; ${ }^{5}$ Medical School, University of Athens, Greece
}

10.1136/lupus-2018-abstract.86

Purpose Next-generation RNA-sequencing was applied to investigate SLE pathogenesis through a comparative transcriptomic analysis of a peripheral lymphoid organ (spleen) and end-organ tissues (kidneys, brain) of lupus-prone and healthy mice.

Methods NZB/W-F1 lupus-prone mice were sacrificed at the pre-puberty, pre-autoimmunity and nephritic stage of the disease. Age-matched C57/BL6 mice were used as controls. Spleen, kidneys and brain were removed and total RNA was extracted. Paired-end RNA-sequencing was performed with Illumina HiSeq 2000 platform. Relative expression levels of transcripts and differentially expressed genes (DEG) (FC >1.5, $p<0.05)$ were calculated. Functional enrichment analysis was performed with IPA, RNEA and gprofiler.

Results To investigate SLE developmental biology, a comparative analysis between the same organs of the same model at different stages of the disease was performed. In the spleen, brain and kidneys of NZB/W-F1 mice, 277, 6 and 8 DEG at the pre-puberty vs pre-autoimmunity stage; 212,6 and 8 DEG at the nephritic vs pre-puberty stage; and 15, 6 and 2 DEG at the nephritic vs preautoimmunity stage were identified, respectively. In the brain, kidneys and spleen, hierarchical clustering revealed 178, 1012 and 2105 genes respectively that were deregulated in at least 1 of 3 stages. Clusters were subjected to functional enrichment analysis. In the brain, genes were mainly downregulated and enriched for metabolic pathways (glycolysis/TCA cycle/Pentose-Phosphate pathway), whereas overexpressed genes were enriched for Jak/Stat signalling pathway. In kidneys, one of the biggest cluster points towards metabolic pathways, particularly to lipid metabolism. In the spleen, DEG were mainly overexpressed. Of note, early genes were particularly enriched in cell-cycle processes; intermediate genes in membrane-related and extracellular matrix functions; and late genes in inflammatory and immune response pathways. To investigate SLE genetic susceptibility, a comparative analysis of the same organ of lupus-prone vs healthy mice at different stages of the disease was performed. In addition to immune response pathways (interferon signalling/antigen-presentation), DEG were involved in canonical pathways such as the phagosome, platelet activation, epithelial adherence junction signalling and the extrinsic prothrombin activation pathway.

Conclusions We identified novel stage-specific tissue-dependent pathways involved in immune response, and tissue injury and response in SLE. Validation is in progress.

\section{PS2:39 GENETIC AND ENVIRONMENTAL RISK FACTORS FOR SYSTEMIC LUPUS ERYTHEMATOSUS - A NATIONWIDE POPULATION STUDY}

1,2 $\mathrm{H}$ Leffers, ${ }^{1} \mathrm{~S}$ Jacobsen, ${ }^{3} \mathrm{H}$ Ullum, ${ }^{4} \mathrm{~T}$ Lange. ${ }^{1}$ Copenhagen Lupus and Vasculitis Clinic, Copenhagen University Hospital, Rigshospitalet, Copenhagen, Denmark; ${ }^{2}$ DANBIO, Copenhagen, Denmark; ${ }^{3}$ Department of Clinical Immunology, Copenhagen University Hospital Rigshospitalet, Copenhagen, Denmark; ${ }^{4}$ Section of Biostatistics, Copenhagen University, Copenhagen, Denmark

\subsection{6/lupus-2018-abstract.87}

Purpose Environmental- and genetic factors' influence in the pathogenesis of rheumatic disease including systemic lupus erythematosus (SLE) have individually been extensively researched. Moreover, a synergistic effect of smoking with anti-citrullinated protein has been established for the risk of developing rheumatoid arthritis. In SLE, however, studies on synergistic effect are few and of limited sample size. We aim to investigate if established genetic end environmental risk factors for SLE display additive or synergistic interactions on the risk of SLE.

Methods A case-control designed nationwide epidemiological study of Danish patients with SLE will be conducted. Patient data-/ 